\title{
The Temperature Dependence of Optical Texture in Chiral Nematic Liquid Crystal Observed by Polarizing Microscope
}

\author{
Risti Suryantari", Flaviana Catherine \\ Department of Physics, Parahyangan Catholic University, Bandung, Indonesia \\ Email address: \\ ristisuryantari@unpar.ac.id (R. Suryantari), flaviana@unpar.ac.id (F. Catherine) \\ ${ }^{*}$ Corresponding author
}

To cite this article:

Risti Suryantari, Flaviana Catherine. The Temperature Dependence of Optical Texture in Chiral Nematic Liquid Crystal Observed by

Polarizing Microscope. Journal of Photonic Materials and Technology. Vol. 6, No. 2, 2020, pp. 21-27. doi: 10.11648/j.jmpt.20200602.12

Received: November 7, 2020; Accepted: November 24, 2020; Published: December 4, 2020

\begin{abstract}
Study the optical-thermal dependence of liquid crystal based on the quantity data of image texture is important to get more information accurately. In this research, the optical-thermal dependence of chiral nematic liquid crystals ink sample was investigated based on the texture and color changes of the sample at various temperatures. The textures of the samples were recorded by the crossed-polarizing optical microscope attached to the hot stage and a camera. The temperature dependence of textures are represented by the hue parameter values taken from the HSV image type. The result obtained from the graph of the increase mean hue parameter against the temperatures was in accordance with the preceding results done by the various techniques. The results show that the higher the temperature of the material, the higher the average hue value. The increase in the hue parameter relates to the color changes of texture from red-dominant to green-dominant. The color difference appeared in various wavelengths represent a diffraction pattern caused by its optically active and the twisted molecular structure. Approaching to the Bragg diffraction equation, the pitch obtained at $0.7 \mu \mathrm{m}$. Furthermore, the relation of the different textures and the twist structure of the sample was evaluated. Considering the sample thickness of $100 \mu \mathrm{m}$ carries out to the conclusion that there are hundreds of twisted nematic liquid crystal molecules in various alignment on a volume space.
\end{abstract}

Keywords: Optical Texture, Temperature, Polarizing Microscope, Chiral Nematic Liquid Crystal, Hue, Diffraction

\section{Introduction}

Liquid crystals are the most common material applied in displays technology industries. Liquid crystals have some anisotropic property as in a crystal. It is possible to combine with other materials, for instance, polymer, chromatic material, and other compounds to achieve some applications such as sensors or actuators $[1,2]$.

Chiral Nematic Liquid Crystal Ink was a compound of nematic liquid crystal type that gives a response by color-play at different temperatures. The result in color shifts indicates that this material has crystal-like optical properties such as birefringence, polarization, and diffraction that are related to the twist-structure and temperature-dependence molecular arrangement. This type has been used successfully in many applications, such as for non-destructive examination in industrial and biomedical fields based on the true-color, known as thermochromic liquid crystal (TLC) [3, 4]. TLC has been applied as a temperature sensor by visualizing the distribution temperature of human body or material surfaces as well [5-7].

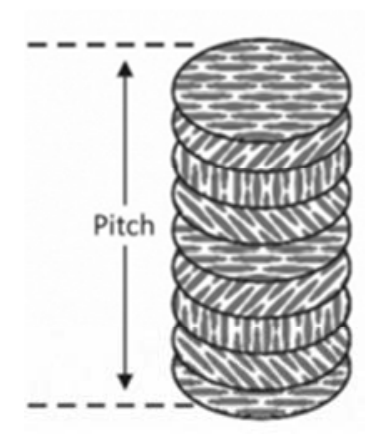

(a)

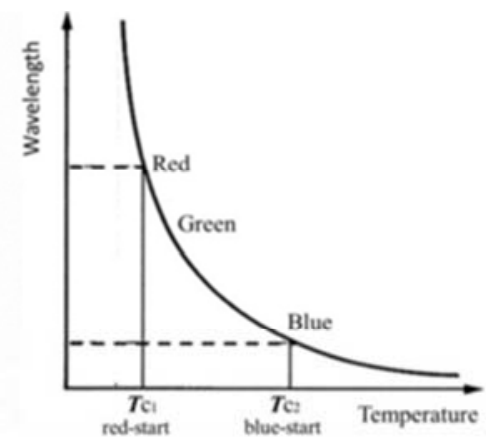

(b)
Figure 1. (a) Molecules alignment of chiral nematic liquid crystal structure. (b) The Graph of reflected wavelengths against the temperatures [3].

The diversity of colors depends on the length of a twisted-length pitch shown in figure 1. The shorter pitch gained at a higher temperature so that the visible light with 
shorter wavelengths would arise. Otherwise, at a lower temperature, a longer pitch formed produces the longer wavelength $[3,4]$.

The color-play will correspond to the color spectrum of visible light, commonly red-green-blue. Figure $1 \mathrm{~b}$ illustrates the reflected wavelengths at various temperatures. In figure $1, T \mathrm{c}$ is the critical temperature when the spesific color starts to appear and changes into another spesific color in the visible light spectrum. $T \mathrm{c}_{1}$ is the lower temperature that representing a red-start and $T \mathrm{c}_{2}$ for higher critical temperature for blue-start [3, 4].

Polarizing microscope is an instrument commonly used to investigate the behavior of liquid crystalline. In a polarizing microscope instrument, an unpolarized light will be converted into polarized light by a polarizer below the sample. This polarizer separates the light source and the sample and can be rotated through $360^{\circ}$. The analyzer is placed above the sample stage and also can be rotatable. By the twisted molecules in various directions of chiral nematic liquid crystals, the lights passing through the sample would be twisted as follows the director of molecules. Then the interference of different rays resulting from the sample generates the contrast image particularly for liquid crystal and non-liquid crystal [8, 9].

In general, there are various techniques for analyzing the microscopy images of liquid crystal. The application of liquid crystals initially was mostly qualitatively, since the difficulty in quantifying color. The development of true-color digital image processing has been resulting in a significant impact on quantitative analysis and temperature measurements. The progress of the image processing techniques by some software has made it possible to develop the real-time temperature measurements using TLC at lower cost [10-14]. An example of software to process the digital image is Matlab.

There are many different ways to specify color. Color is often considered as a combination of the primary colors, red, green, and blue (RGB). Those colors can be combined to produce millions of colors in the visible spectrum. RGB color has three components and each component values at 0-255 which means more complicated in the analysis because of many numbers involved. Color image in RGB type is more complex than in monochromatic type. By converting the RGB images to monochromatic images digitally affects the process of processing data such as the possibility in taking less time in running a computer program [15].

The other example of an image typically is hue-saturation-intensity (HSI) or hue-saturation-value (HSV) data [15]. The HSV color space is appropriate with the color perception of humans better than the RGB color space and simpler values from 0 to 1 . In Matlab, the functions rgb2hsv convert RGB images to HSV [16].

Hue Colors range vary as follows the color-wheel from red through yellow, green, cyan, blue, magenta, and back to red as shown in figure 2 . It has values from 0 to 1 , meaning that the corresponding colors change from red to blue. The increase of hue related to the different color rotates $360^{\circ}$ from red to violet as shown in the HSV color space in figure 2. While saturation varies from 0 to 1 , corresponding colors specified by hues vary from unsaturated to fully saturated, in the term of shades of gray. As the value component of HSV varies from 0 to 1 , meaning that the corresponding colors become increasingly brighter from 0 to 1 . The figure below illustrates the HSV color space $[15,16]$.

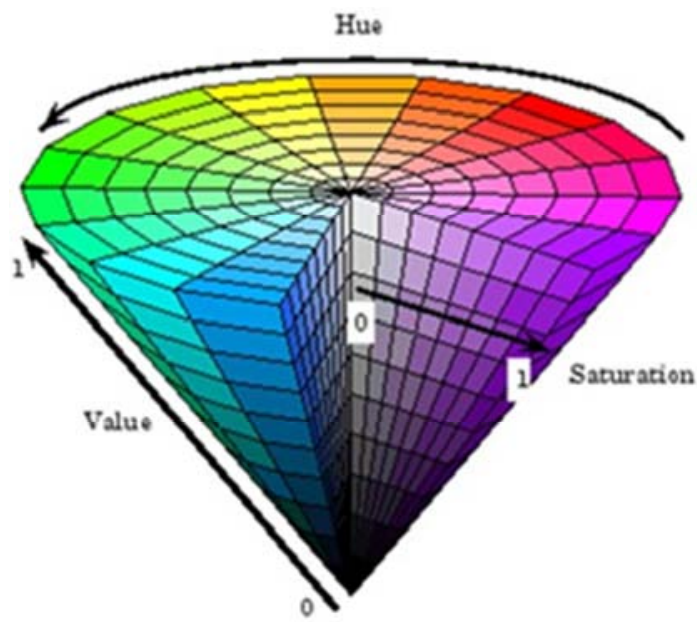

Figure 2. Illustration of the HSV Color Space.

Considering the chiral nematic liquid crystal ink color characteristic and the compatibility with the hue color space in the above figure illustrated, hues make a transition from low to high values, starting at red color to blue color. The color in deep blue appearance has the highest value, while the deep red color has the lowest value [16].

In this work, the optical-thermal dependence of chiral nematic liquid crystals ink samples will be investigated based on the texture and color changes of the sample at various temperatures. The sample observed by the crossed- polarizing optical microscope attached to the hot stage and a camera. The texture and color changes will be analyzed by the hue parameter taken from HSV image type.

\section{Method}

The chiral nematic liquid crystal ink sample was arranged between two glasses spaced by a $100 \mu \mathrm{m}$ spacer without any treatment in orientational order. The sample was observed under a crossed polarizing optical microscope attached to hot stage temperature control. The sample has a bandwidth of $24^{\circ} \mathrm{C}$ to $29^{\circ} \mathrm{C}$. It means that the sample starts to change at $24^{\circ} \mathrm{C}$ and completes its change at $29^{\circ} \mathrm{C}$.

The color images captured by the camera represented the true color pixel tone that ranges from 0 to 255 in RGB color. To simplify, the RGB images of each temperature were converted to monochromatic images using Matlab. We converted the RGB images to HSV (hue, saturation, value) images by $r g b 2 h s v$ function and took the hue parameter only that represented the predominant colors of the images in the range of 0 to 1 [16]. To analyze the optical properties by different texture and color, we concern with the mean statistical of hue parameter to illustrate the behavior of color difference based on the graph of mean hue parameter correlated to the increasing temperatures. 


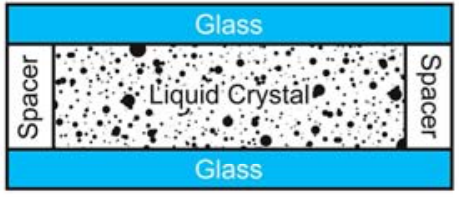

(a)

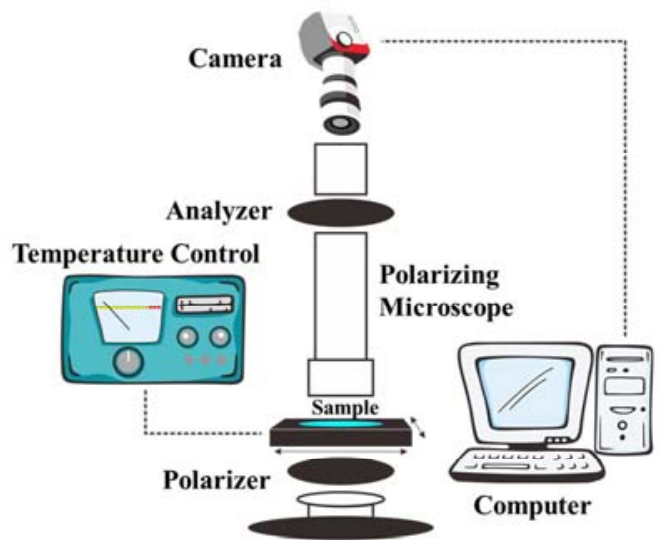

(b)

Figure 3. (a) Side view of sample. (b) Instrumentation set-up.

Next, we correlated the result with the increasing green component value of the RGB image, by the forethought that the increasing of hue results from increasing the green color at exceeding the critical temperature. Finally, we implemented an im-profile technique along line segmentation of crossed-pattern to investigate the diffraction phenomenon of TLC [16].

\section{Results and Discussions}

In this experiment, we used a hot stage to control the temperature from $27^{\circ} \mathrm{C}$ to $36^{\circ} \mathrm{C}$. The image of each temperature was captured by a crossed polarizing optical microscope. In the crossed polarizing optical microscope, the polarizer and the analyzer arranged to form an angle of 90 degrees $[8,9]$. Because of the anisotropic property of liquid crystal, and the molecular structures in chiral nematic, the polarized light passing through the sample will be twisted following the twist alignment of the molecules [2-4].

Moreover, the un-oriented alignment of the sample causes the various directions of waves entering the sample. On the other hand, the waves pass through the sample in different phases and because of constructive and destructive interferences, they produce reflected rays in various directions passed through the analyzer. It leads to a high-contrast image and shapes some patterns depend on the temperatures. Those reflected lights are not monochromatic and depend on many factors in modifying the resulting color information, for instance the refractive index of the sample, the surrounding temperatures, the angle of observation, the resolution of the camera, the light source of the instrument, and the surrounding light intensity $[3,8]$.

A result shown by recorded images illustrates that no significant different texture from $26^{\circ} \mathrm{C}$ to $30^{\circ} \mathrm{C}$. The color appears in the same yellow dominant color. The predominant color may have been affected by the source light used in the microscope instrument. At $31^{\circ} \mathrm{C}$, the texture has begun to create a unique shape, different as previously. Then at $32^{\circ} \mathrm{C}$, a new texture is formed by a cross-like texture [17]. Exceed this temperature, the texture remains constant, with no significant change.

Figures $4 \mathrm{a}$ and $4 \mathrm{~b}$ show visualization of the sample at a temperature of $31^{\circ} \mathrm{C}$ and $32^{\circ} \mathrm{C}$, when the extreme change occurred clearly. The most significant change occurs at a specific temperature which is the critical temperature, which is characterized by a difference in texture into a cross-shape and the appearance of colors following the diffraction pattern from reddish to greenish repeatedly on the polar cross.

This critical temperature depends on the range of active temperature of the sample. Here the sample has bandwidth at $26^{\circ} \mathrm{C}$ to $29^{\circ} \mathrm{C}$, means that the color starts to appear at $26^{\circ} \mathrm{C}$, and stable at $29^{\circ} \mathrm{C}$ or more until the clearing point temperature. Exceed the clearing point temperature, no color appears. According to the experiment result, the critical temperature is obtained at $31^{\circ} \mathrm{C}$ when the texture begins to change to a stable cross-like texture. The difference in critical temperatures and the specification temperature of the material may be caused by the uncertainty of temperature measurement. It may be affected by some factors, such as the stabilization of hot stage temperature and the thickness of the sample. Moreover, the more thickness of the sample may cause a longer time of the heat distribution [2].

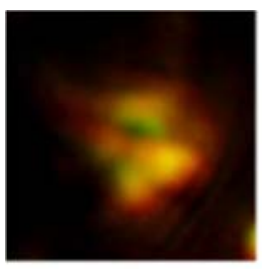

(a)

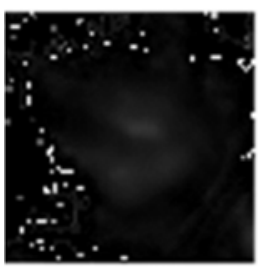

(c)

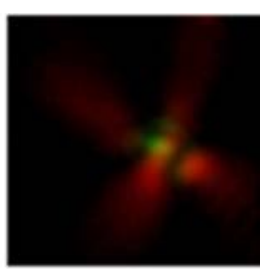

(b)

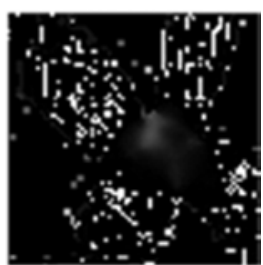

(d)
Figure 4. The RGB images of chiral nematic sample at (a) $31^{\circ} \mathrm{C}$ (b) $32^{\circ} \mathrm{C}$, and the hue images of chiral nematic sample at (a) $31^{\circ} \mathrm{C}$ (b) $32^{\circ} \mathrm{C}$, observed by polarizing microscope. 
The change of color is represented by the hue parameter quantitatively [18]. Figures $4 \mathrm{c}$ and $4 \mathrm{~d}$ illustrate the hue image for $31^{\circ} \mathrm{C}$ and $32^{\circ} \mathrm{C}$ samples, respectively. Then, the average hue value is executed for each image. Statistical data taken from Matlab shows that higher temperatures resulting in a higher average hue value (mean hue)

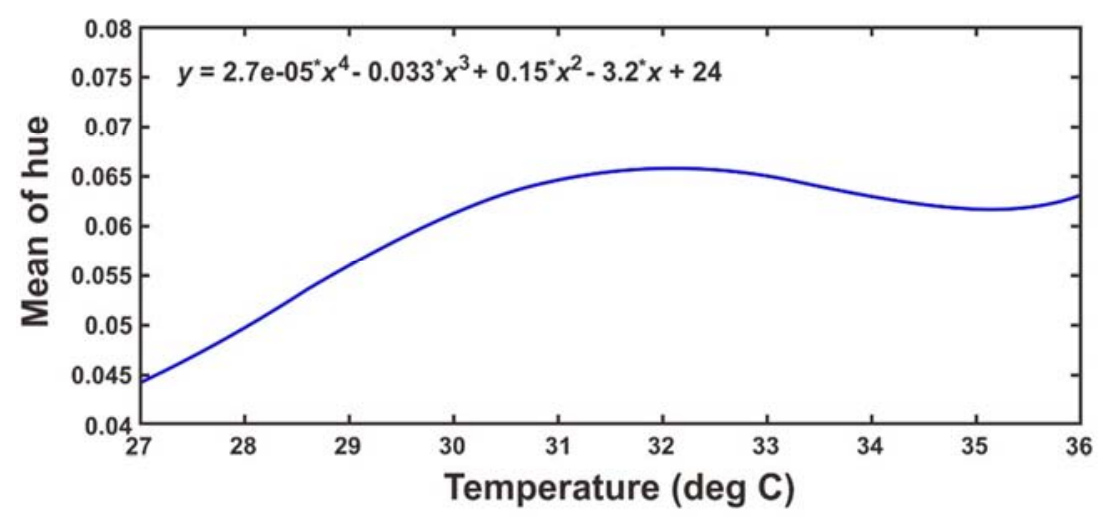

Figure 5. The graph of the mean hue component of HSV image against the increasing temperatures and high-order polynomial fits.

As shown in figure 5, the data are illustrated by fitting a fourth-degree polynomial to establish a smooth huetemperature relation. The result obtained from the increasing mean hue parameter value by increasing the temperature as shown in figure 5 is in accordance with the preceding results done by the various techniques [18].

As an illustration in figure 5 , in the range of $26^{\circ} \mathrm{C}$ to $31^{\circ} \mathrm{C}$, the mean-hue increase gradually, and tend to constant from $31^{\circ} \mathrm{C}$ to $36^{\circ} \mathrm{C}$. It is appropriate with the images, where from $31^{\circ} \mathrm{C}$ to $36^{\circ} \mathrm{C}$ the texture remains constant as shown in figure $4 \mathrm{~b}$. Compared with Figures $4 \mathrm{a}$ and $4 \mathrm{~b}$, the increase of the mean hue is caused by the appearance of more green color. Figure $4 \mathrm{~b}$ illustrates the dominant green color appears at the center of the cross-like texture.
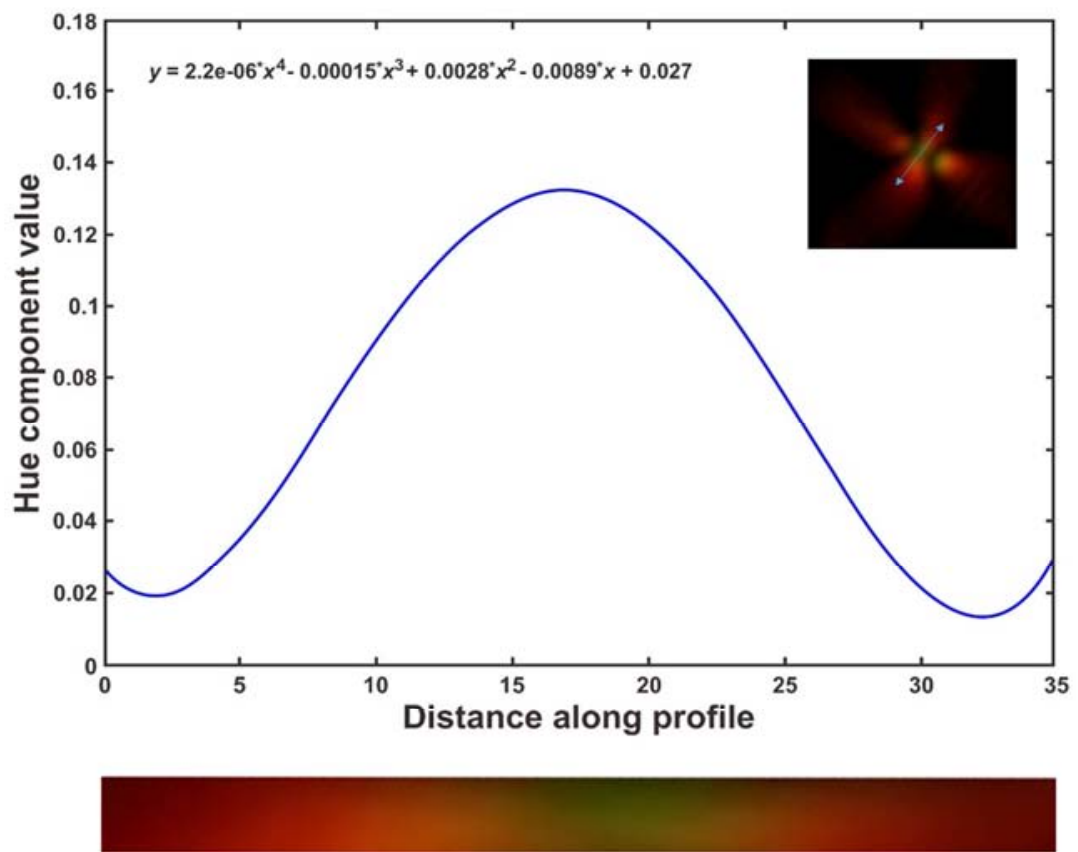

Figure 6. The graph of hue component value of HSV image against the distance along profile at $32^{\circ} \mathrm{C}$. The peak of the graph represents the center of the profile picture illustrated below the graph.

Next, we analyze the different arise colors along with the cross-like texture. By taking hue values along line segmentation using the im-profile method in Matlab, we get the result as shown in figure 6 . The graph in figure 6 represents the different hue values and reaches the peak at the center of line segmentation, which is the center or cross-like texture as shown in the picture below the graph. The color of cross-like texture follows the un-fully diffraction pattern, from red to green in the center, and back to red again at the end of a cross-like texture. Figure 6 also visualizes that the red color has the lowest hue value, and the green, here in the center of line segmentation, has the highest hue value.

The graph appropriate with the images result, as previous, explanation that the increasing of the mean hue is caused by the appearance of increasing the green color. For further information, by taking the value of the green component in the 
RGB image along line segmentation similar to in figure 6 , it can be shown the similarity graph behavior corresponding to the hue graph as illustrated in figure 7 . Figure 7 proves that the increase of mean hue in the figure 5 is carried out by raising the green color of texture because of increasing the temperature.

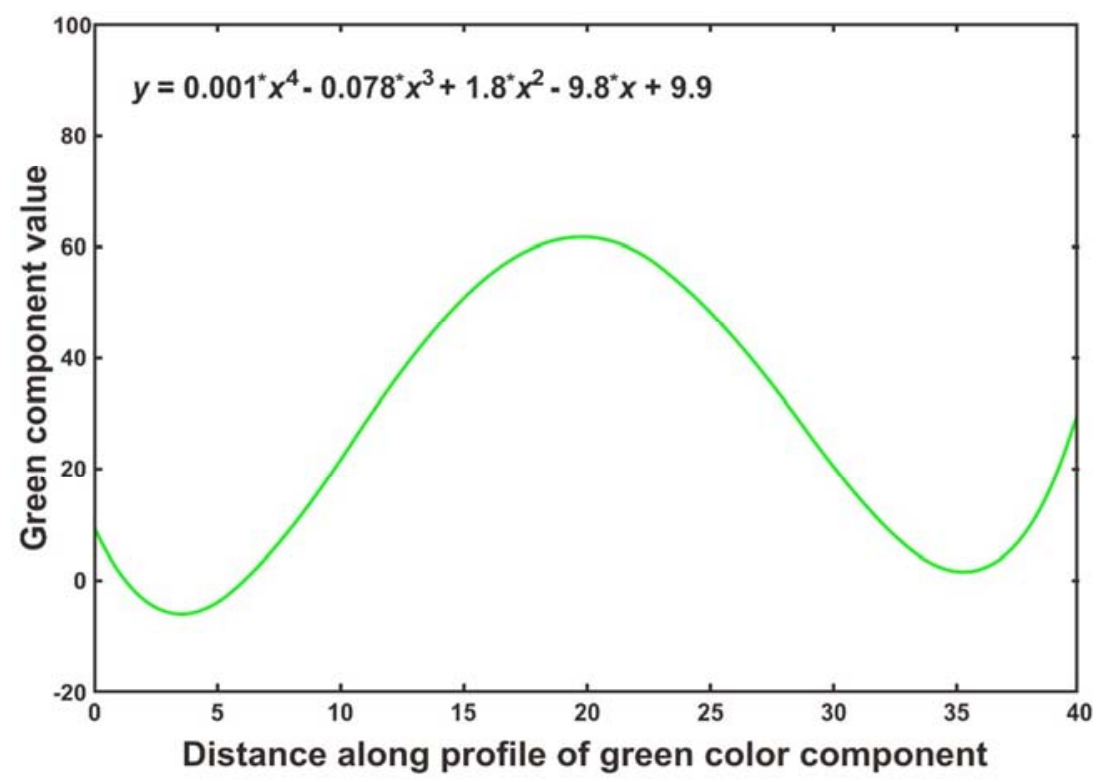

Figure 7. The graph of green component value of RGB image against the distance along profile at $32^{\circ} \mathrm{C}$. The peak of the graph represents the center of the profile picture illustrated in figure 6 .

Furthermore, the relation of the different textures and the twist structure of the sample visible light spectrum along a cross-like pattern would be evaluated by measuring the pitch length first. Based on the visible light spectrum along with a cross-like pattern as shown in figure 8 , on either side of the central maximum lie the first-order maxima. The first-order color appearing here is green (shortest wavelength) that comes first (at the smallest angle), and red is last. The colors do not progress through the pure spectral colors and it is not complete as a visible light spectrum because of the characteristic of the sample that is only possible to produce the red-green-blue color of the range of its active temperature.

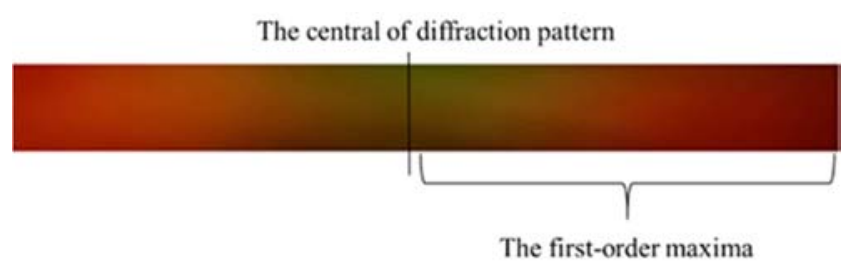

Figure 8. The diffraction pattern at $32^{\circ} \mathrm{C}$.

Based on the color in figure 8, the various colors appear in a diffraction-like pattern. The twist and periodic layered structures of chiral nematic liquid crystal allow it to behave like a three-dimensional diffraction grating and reflects the wavelengths in the visible spectrum. This principle of how the light could be reflected in a crystal similar to the Bragg scattering that is observed in crystalline solids with x-rays wavelength. Therefore, the magnitude of the reflected wavelength as shown in figure 8 can be used to determine the pitch-length approached by the principle of the Bragg scattering. This Bragg-type scattering gives rise to the characteristic of iridescent colors as shown in figure 9.

The pitch of the materials is determined using the approaching Bragg diffraction equation. The relation between wavelength reflected and the angle of the incident beam normal to the surface is approximately described by equation $1[1,3]$.

$$
2 d \sin \theta=m \lambda
$$

Where $d$ is Bragg layers distance, $\theta$ is the normal angle that comes to the surface of the chiral nematic liquid crystal, and $\lambda$ is the reflected wavelength. $2 d$ is equal to the length of the pitch, $p[1,3,19]$.

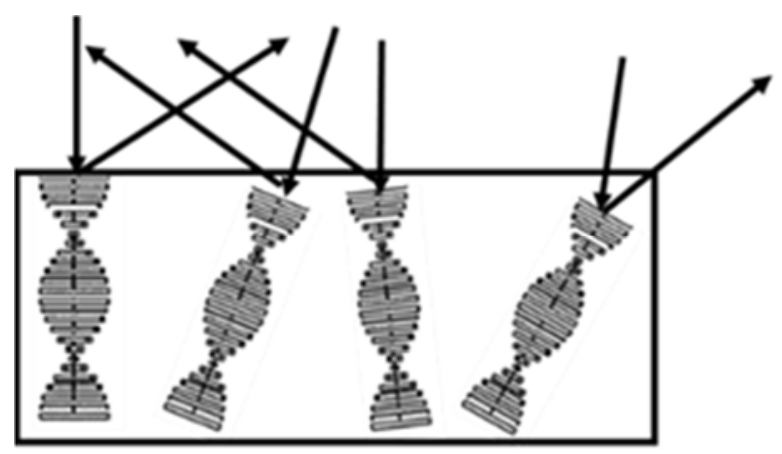

Figure 9. Bragg scattering illustration in chiral nematic [3].

The last color we see in figure 8 at either extreme $\left(\theta= \pm 90^{\circ}\right)$ is red. Thus, the first-order maximum for red light occurs at $\pm 90^{\circ}$. The red wavelength is $\pm 700 \mathrm{~nm}$. By approaching the diffraction equation as equation (1), the $2 d$ or a pitch $(p)$ is calculated at $0.7 \mu \mathrm{m}$ approximately, and the half of pitch at $0.35 \mu \mathrm{m}$ approximately [19]. 


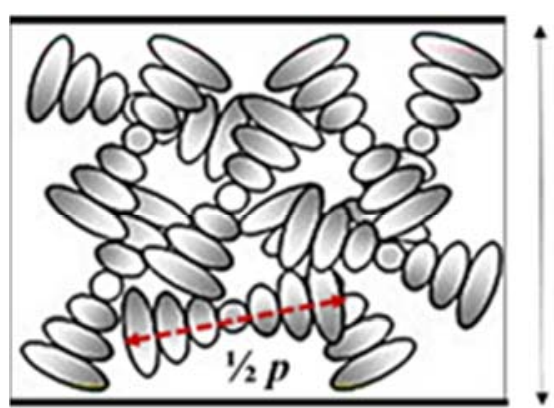

$100 \mu \mathrm{m}$

Figure 10. Side view of oblique alignment in chiral nematic sample.

In this experiment, the thickness of the sample is $100 \mu \mathrm{m}$, so that there are almost a hundred twisted nematic liquid crystals along the vertical axis of sample thickness. However, because in this experimental method we did not arrange the molecular alignment of the sample, the illustration of molecular alignment to show the comparison of the thickness and the pitch can be illustrated as in figure 10 [20]. In figure 10, the scale is not being illustrated well. The ratio of the half of pitch and the sample thickness should be obtained in a very small value at 0.0035 .

\section{Conclusions}

In summary, based on the images recorded by a crossed polarizing microscope, the texture has begun to shape a unique shape at $31^{\circ} \mathrm{C}$, the new texture is formed by a cross-like texture. Exceed $32^{\circ} \mathrm{C}$, the texture remains constant, with no significant change, appropriate with the characteristic of active temperature of the sample. The result obtained from the graph of mean hue against the temperature, indicates the increase mean hue by increasing the temperature appropriate with earlier published literature done by the different techniques.

The increase of the mean hue is caused by the appearance of more green color that be illustrated the dominant green color appears at the center of the cross-like texture. By taking hue values along line segmentation using the im-profile method in Matlab, and presenting in a graph, it shows the different hue values obviously, and the peak hue is achieved at the center of line segmentation, which is the center or cross-like texture. Moreover, the color of cross-like texture follows the un-fully diffraction pattern, and by a simple calculation using an approaching Bragg diffraction equation, it is obtained a pitch at $\pm 0.7 \mu \mathrm{m}$. Considering the sample thickness of $100 \mu \mathrm{m}$ carries out to the conclusion that there are hundreds of twisted nematic liquid crystal molecules in various alignment on a volume space.

\section{Acknowledgements}

This study was supported by the Research Development Institution of Parahyangan Catholic University. The facilities of experiment were supported by Material Laboratory, Sciences and Mathematics Faculty, Gadjah Mada University, Indonesia.

\section{References}

[1] S. Chandrasekhar, Liquid Crystal, New York: Cambridge University Press, 1992.

[2] Yang, Deng-Ke \& Wu, Shin-Tson, Fundamentals of Liquid Crystal Devices, John Wiley \& Son Ltd, 2006.

[3] Hallcrest, Handbook of Thermochromic Liquid Crystal Technology, Glenview, IL: Hallcrest., 1991.

[4] Ian Sage. (2011). Thermochromic liquid crystals, Liquid Crystals, 38: 11-12, 1551-1561.

[5] Shuang Zhou. (2018). Recent progresses in lyotropic chromonic liquid crystal research: elasticity, viscosity, defect structures, and living liquid crystals, Liquid Crystals Today, 27: 4, 91-108.

[6] David Coates. (2015). Development and applications of cholesteric liquid crystals, Liquid Crystals, 42: 5-6, 653-665.

[7] Stasiek, J., Kowalewski, Tomasz. (2002). Thermochromic liquid crystals in heat transfer research, Proceedings of SPIE The International Society for Optical Engineering, 10 (1), 110.

[8] Nastishin, Yu A., et al. (2005). Optical characterization of the nematic lyotropic chromonic liquid crystals: Light absorption, birefringence, and scalar order parameter. Physical Review, E 72.4: 041711 .

[9] Smalyukh, Ivan I., S. V. Shiyanovskii, and O. D. Lavrentovich. (2001). Three-dimensional imaging of orientational order by fluorescence confocal polarizing microscopy. Chemical Physics Letters, 336.1-2: 88-96.

[10] Sigaki Higor, Y. D., Lenzi, E. K., Zola, R. S., Matjaž, P., \& Ribeiro, H. V. (2020). Learning physical properties of liquid crystals with deep convolutional neural networks. Scientific Reports (Nature Publisher Group), 10 (1).

[11] B. T. P. Madhav, P. Pardhasaradhi, R. K. N. R. Manepalli \& V. G. K. M. Pisipati. (2015). Histogram equalization technique to analyse induced cholesteric phase in nanodoped liquid crystalline compound. Liquid Crystals, 42: 7, 989-997.

[12] K. Bjorknas, M. A. Geday \& E. P. Raynes. (2003). Quantitative conoscopic imaging of cholesteric thin films. Liquid Crystals, 30: $8,889-897$.

[13] S. Sreehari Sastry, K. Mallika, B. Gowri Sankara Rao, Ha Sie Tiong \& S. Lakshminarayana. (2012). Identification of phase transition temperatures by statistical image analysis, Liquid Crystals, 39: 6, 695-700.

[14] S. Sreehari Sastry, S. T. Ha, B. Gowri Sankara Rao, K. Mallika \& T. Vindhya Kumari. (2012). Optical properties of a mesogen by image analysis, Liquid Crystals, 39: 11, 1414-1419.

[15] Gonzales, R. C., Woods, R. E., Digital Image Processing, 2ed, Prentice Hall, 2002.

[16] Information on https://www.mathworks.com/help.

[17] Tanaka, S., Yoshida, H., Kawata, Y. et al. (2015). Double-twist cylinders in liquid crystalline cholesteric blue phases observed by transmission electron microscopy. Sci Rep 5, 16180. 
[18] Bharara, Manish, Liquid Crystal Thermography in Neuropathic Assessment of Diabetic Foot, PhD Thesis, Bournemouth University, 2007.

[19] Ingo Dierking, Textures of Liquid Crystals, Wiley-VCH Verlag $\mathrm{GmbH} \& \mathrm{Co} . \mathrm{KGaA}, 2003$.
[20] O. Lavrentovich. (2020). Electromagnetically tunable cholesterics with oblique helicoidal structure. Opt. Mater. Express 10, 2415-2424. 